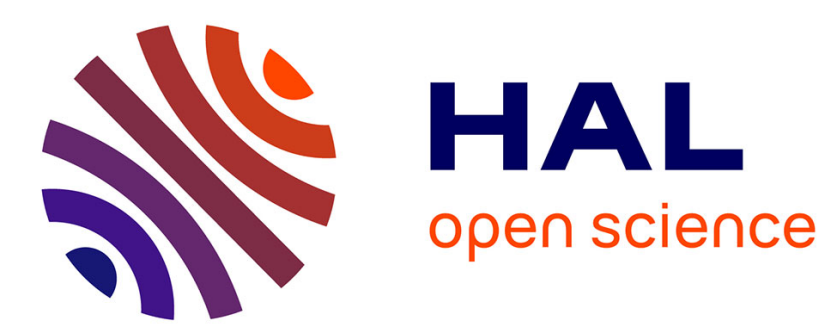

\title{
Sum-of-Product Architectures Computing Just Right
}

Florent de Dinechin, Matei Istoan, Albdelbassat Massouri

\section{To cite this version:}

Florent de Dinechin, Matei Istoan, Albdelbassat Massouri. Sum-of-Product Architectures Computing Just Right. ASAP - Application-specific Systems, Architectures and Processors, Jun 2014, Zurich, Switzerland. hal-00957609

\section{HAL Id: hal-00957609 \\ https://hal.inria.fr/hal-00957609}

Submitted on 10 Mar 2014

HAL is a multi-disciplinary open access archive for the deposit and dissemination of scientific research documents, whether they are published or not. The documents may come from teaching and research institutions in France or abroad, or from public or private research centers.
L'archive ouverte pluridisciplinaire HAL, est destinée au dépôt et à la diffusion de documents scientifiques de niveau recherche, publiés ou non, émanant des établissements d'enseignement et de recherche français ou étrangers, des laboratoires publics ou privés. 


\title{
Sum-of-Product Architectures Computing Just Right
}

\author{
F. de Dinechin, Matei Istoan, Abdelbasset Massouri \\ Université de Lyon, INRIA, \\ INSA-Lyon, CITI-INRIA, F-69621, Villeurbanne, France
}

\begin{abstract}
Many digital filters and signal-processing transforms can be expressed as a sum of products with constants (SPC). This paper addresses the automatic construction of low-precision, but high accuracy SPC architectures: these architectures are specified as last-bit accurate with respect to a mathematical definition. In other words, they behave as if the computation was performed with infinite accuracy, then rounded only once to the low-precision output format. This eases the task of porting double-precision code (e.g. Matlab) to low-precision hardware or FPGA. The paper further discusses the construction of the most efficient architectures obeying such a specification, introducing several architectural improvements to this purpose. This approach is demonstrated in a generic, open-source architecture generator tool built upon the FloPoCo framework. It is evaluated on Finite Impulse Response filters for the ZigBee protocol.
\end{abstract}

\section{INTRODUCTION}

This article addresses the implementation of digital filters and other signal-processing transforms that can be expressed as a sum of products with constants (SPC). Specifically, a SPC is any computation of the form

$$
y=\sum_{i=0}^{N-1} a_{i} x_{i}
$$

where the $a_{i}$ are real constants and the $x_{i}$ are inputs in some machine-representable format.

Examples of such computations include FIR and IIR filters, and classical signal processing operators such as the discrete cosine transform (DCT).

Equation (1), along with a mathematical definition of each $a_{i}$, constitute the mathematical specification of the problem. To specify an implementation, we need to define finite-precision input and output formats. More subtly, we also need to specify the accuracy of the computation. In classical design methodologies, these are separate concerns, although there is some obvious link between them. For instance, in the implementation process, the real-valued $a_{i}$ must be rounded to some machine format. This format obviously depends on the input and output formats, and obviously impacts the computation accuracy.

A first contribution of this article is to explicit this link between I/O precision and accuracy, based on the following claim: an architecture should be last-bit accurate, i.e. return only meaningful bits. This simple specification, which will be formalized in the body of the article, enables

- a very simple interface (Fig. 1) to an implementation tool, focusing designer's freedom on those design

This work is partly supported by the MetaLibm project of the french Agence Nationale de la Recherche (ANR)

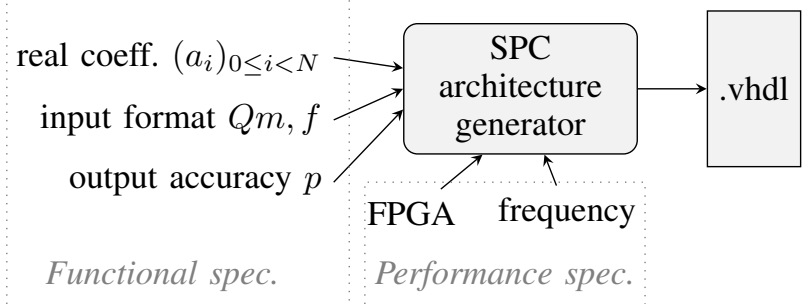

Fig. 1. Interface to the proposed tool.

parameters which are relevant. For instance, it will be shown that the format to which an $a_{i}$ will be rounded is not a relevant design parameter: it can be deduced optimally from the mathematical specification on one side, and the input/output formats on the other side.

- a fully automated implementation process that builds provably-minimal architectures of proven accuracy.

A second contribution is an actual open-source tool that demonstrates this implementation process. This tool automatically generates VHDL for SPC computations. Built upon the FloPoCo project ${ }^{1}$, it also benefits from the FloPoCo backend framework. The generated architectures are optimized for a user-specified FPGA target running at a user-specified frequency (Fig. 1).

A third contribution of this work is to introduce several architectural novelties. The constant multipliers are built using an evolution of the KCM algorithm [1], [2] that manages multiplications by a real constant [3]. The summation is efficiently performed thanks to the BitHeap framework recently introduced in FloPoCo [4]. These technical choices lead to logic-only architectures suited even to low-end FPGAs, a choice motivated by work on implementing the ZigBee protocol standard [5] (the examples illustrating this article are from this standard). However, the same philosophy could be used to build other architecture generators, for instance exploiting embedded multipliers and DSP blocks.

\section{LAST-BIT ACCURACY: DEFINITIONS AND MOTIVATION}

\section{A. Fixed-point formats, rounding errors, and accuracy}

Technically, the overall error of a SPC architecture in fixedpoint is defined as the difference between the computed value $\widetilde{y}$ and its mathematical specification:

$$
\epsilon=\widetilde{y}-y=\widetilde{y}-\sum_{i=0}^{N-1} a_{i} x_{i}
$$

\footnotetext{
${ }^{1}$ http://flopoco.gforge.inria.fr/
} 
In all the article, we try to use tilded letters (e.g. $\widetilde{y}$ above) for approximate or rounded terms. This is but a convention, and the choice is not always obvious. For instance, here, the $x_{i}$ are fixed-point inputs, and most certainly the result of some approximate measurement or computation. However, from the point of view of the SPC, they are inputs, they are given, so they are considered exact.

In all the following, we note

- $\bar{\epsilon}$ the error bound, i.e. the maximum of the absolute value of $\epsilon$,

- $\quad p$ the precision of the output format, such that $2^{-p}$ is the value of the least-significant bit (LSB) of $\widetilde{y}$;

- $\quad o_{p}(x)$ the rounding of a real $x$ to the nearest fixedpoint number of precision $p$.

This rounding, in the worst case, entails an error $\left|\circ_{p}(x)-x\right|<2^{-p-1}$. For instance, rounding a real to the nearest integer $(p=0)$ may entail an error up to $0.5=2^{-1}$. This is a limitation of the format itself. Therefore, the best we can do, when implementing (1) with a precision- $p$ output, is a perfectly rounded computation with an error bound $\bar{\epsilon}=2^{-p-1}$.

Unfortunately, since the $a_{i}$ are arbitrarily accurate, reaching perfect rounding accuracy may require arbitrary intermediate precision. This is not acceptable in an architecture. We therefore impose a slightly relaxed constraint: $\bar{\epsilon}<2^{-p}$. We call this last-bit accuracy, because the error must be smaller than the value of the last (LSB) bit of the result. It is sometimes called faithful rounding in the literature.

Considering that the output format implies that $\bar{\epsilon} \geq 2^{-p-1}$, it is still a tight specification. For instance, if the exact $y$ happens to be a representable precision- $p$ number, then a lastbit accurate architecture will return exactly this value.

The main reason for chosing last-bit accuracy over perfect rounding is that, as will be shown in Section III, it can be reached with very limited hardware overhead. Therefore, an architecture that is last-bit-accurate to $p$ bits makes more sense than a perfectly rounded architecture to $p-1$ bits, for the same accuracy $2^{-p}$.

\section{B. Application-specific architectures should be last-bit accu- rate}

The rationale for last-bit accuracy architectures is therefore as follows. On the one hand, last-bit accuracy is the best that the output format allows at acceptable hardware cost. On the other hand, any computation less than last-bit accurate is outputting meaningless bits in the least significant places. If we are designing an architecture, this should clearly be avoided: each output bit has a cost, not only in routing, but also in power and resources downstream, in whatever will process this bit. This price should be paid only for those bits that hold useful information.

\section{Real constants for highest-level specification}

It is important to emphasize that the $a_{i}$ constants are considered in this work as infinitely accurate real numbers.

In many cases, the coefficients are defined in textbooks by explicit formulas. This is the case for the DCT, but also for classical signal-processing filters, such as the ones that motivated this work: the half-sine FIR, and the root-raised cosine FIR [5]. In such cases, last-bit accuracy is defined with respect to this purely mathematical specification. Thus the implementation will be as faithful to the maths as the $\mathrm{I} / \mathrm{O}$ formats allow.

In other cases, we do not have explicit formula, but the coefficients are provided (e.g. in Matlab) as double-precision numbers. In such cases, last-bit accuracy is defined with respect to an infinitely accurate evaluation of (1) using exactly the values provided for the coefficients. In practice, this means that the architecture will behave numerically as if (1) was evaluated by Maple in double precision (or better), with a single rounding of the final result to the output fixed-point format.

When converting a mathematical or Matlab specification to low-precision hardware, last-bit accuracy thus ensures that the hardware is as close to the specification as possible. In addition, it also considerably eases the conversion process itself. Indeed, in a classical design flow, such a conversion involves steps such as coefficient quantization and datapath dimensioning, which have to be decided by the designer. This requires a lot of effort, typically by trial-and-error until a small enough implementation with a good enough signal-noise ratio is attained. In our approach, as the sequel will show, optimal choices for these steps can be deduced from the specification. Freeing the designer from these time-consuming and errorprone tasks may be the main contribution of this work. It is definitely a progress with respect to most existing DSP tools.

\section{Tool interface}

To sum up, the ideal interface (Fig. 1) should

- allow a user to input the $\left(a_{i}\right)_{0 \leq i<N}$ to the tool either as mathematical formulas, or as arbitrary-precision floating-point numbers. Our current implementation only supports the latter so far;

- $\quad$ offer one single integer parameter $p$, to serve both as accuracy specification and as the definition of the LSB of the output format.

Let us now show that the MSB of the output format, as well as all the internal precision parameters, may be deduced from the parameters depicted on Fig. 1.

\section{ENSURING LAST-BIT ACCURACY}

The summation of the various terms $a_{i} x_{i}$ is depicted on Fig. 2. For this figure, we take as an example a 4-tap FIR with arbitrary coefficients. As shown on the figure, the exact product of a real $a_{i}$ by an input $x_{i}$ may have an infinite number of bits.

\section{A. Determining the most significant bit of $a_{i} x_{i}$}

A first observation is that the MSB of the products $a_{i} x_{i}$ is completely determined by the $a_{i}$ and the input fixed-point format. Indeed, we have fixed-point, hence bounded, inputs. If the domain of $x_{i}$ is $x_{i} \in(-1,1)$, the MSB of $a_{i} x_{i}$ is the MSB of $\left|a_{i}\right|$. This is illustrated by Figure 2. In general, if $\left|x_{i}\right|<M$, the MSB of $a_{i} x_{i}$ will be $\left\lceil\log _{2}\left(\left|a_{i}\right| M\right)\right\rceil$. 


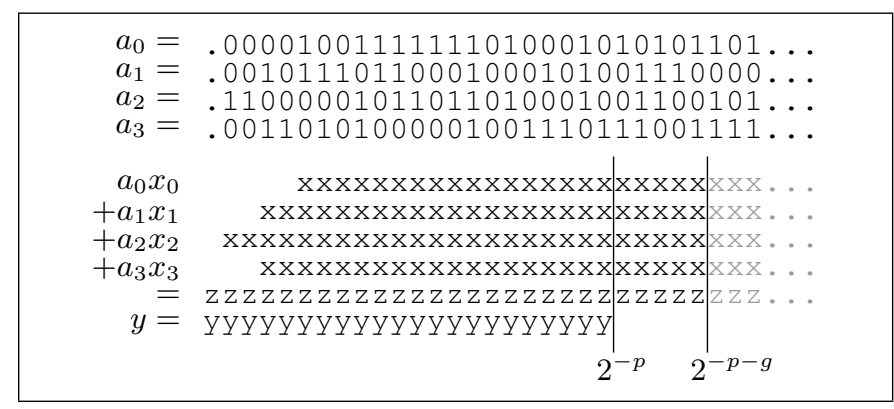

Fig. 2. The alignment of the $a_{i} x_{i}$ follows that of the $a_{i}$

This is obvious if $a_{i} x_{i}$ is positive. It is not true if $a_{i} x_{i}$ is negative, since in two's complement it needs to be signextended to the MSB of the result. However the sign extension of a signed number 00...00sxxxx, where $\mathrm{s}$ is the sign bit, may be performed as follows:

$$
\begin{array}{r}
00 \ldots 0 \overline{\mathrm{s} x x x x x x x} \\
+\quad 11 \ldots 110000000 \\
=\quad \text { s...ss }
\end{array}
$$

Here $\bar{s}$ is the complement of $\mathrm{s}$. The reader may check this equation in the two cases, $s=0$ and $s=1$. Now the variable part of the product has the same MSB as $\left|a_{i}\right|$.

This transformation is not for free: we need to add a constant. Fortunately, in the context of a summation, we may add in advance all these constants together. Thus the overhead cost of two's complement in a summation is limited to the addition of one single constant. In many cases, as we will see, this addition can even be merged for free in the computations of the $a_{i} x_{i}$.

\section{B. Determining the most significant bit of the result}

A second observation is that the MSB of the result is also completely determined by the $\left(a_{i}\right)_{0<i<N}$. Again from the bound $\left|x_{i}\right|<M$, we may deduce that the MSB of the result is

$$
\operatorname{MSB}_{\widetilde{y}}=\left\lceil\log _{2}\left(\sum_{i=0}^{N-1}\left|a_{i}\right| M\right)\right\rceil .
$$
occur.

This upper bound guarantees that no overflow will ever

For this reason, we do not need the user to specify the output MSB on Figure 1: providing the $a_{i}$ is enough.

In some cases, however, the context may dictate a tighter bound due to some additional relationship between the $x_{i}$, for instance a consequence of their being successive samples of the same signal. For this reason, the tool offers the option of specifying completely the output format, but this is only an option. Otherwise, the tool will use Eq. (3) to compute the minimal value of the output MSB that guarantees the absence of overflow.

\section{Determining the least significant bit: error analysis}

As we have seen, a single integer $p$, the weight of the least significant bit (LSB) of the output, also specifies the accuracy of the computation.
However, performing all the internal computations to this precision would not be accurate enough. Suppose for instance that we could build perfect hardware constant multipliers, returning the perfect rounding $\widetilde{p}_{i}=o_{p}\left(a_{i} x_{i}\right)$ of the mathematical product $a_{i} x_{i}$ to precision $p$. Even such a perfect multiplier would entail an error, defined as $\epsilon_{i}=\widetilde{p}_{i}-a_{i} x_{i}$, and bounded by $\epsilon_{i}<\overline{\epsilon_{\text {mult }}}=2^{-p-1}$. Again this rounding error is the consequence of the limited-precision output format. The problem is that in a SPC, such rounding errors add up. Let us analyze this.

The output value $\widetilde{y}$ is computed in an architecture as the sum of the $\widetilde{p}_{i}$. This summation, as soon as it is performed with adders of the proper size, will entail no error. Indeed, fixed-point addition of numbers of the same format may entail overflows (these have been taken care of above), but no rounding error. This enables us to write

$$
\widetilde{y}=\sum_{i=0}^{N-1} \widetilde{p}_{i},
$$

therefore

$$
\epsilon=\sum_{i=0}^{N-1} \widetilde{p}_{i}-\sum_{i=0}^{N-1} a_{i} x_{i}=\sum_{i=0}^{N-1} \epsilon_{i} .
$$

Unfortunately, in the worst case, the sum of the $\epsilon_{i}$ can come close to $N \overline{\epsilon_{\text {mult }}}$, which, as soon as $N>2$, is larger than $2^{-p}$ : this naive approach is not last-bit accurate.

The solution is, however, very simple. A slightly larger intermediate precision, with $g$ additional bits ("guard" bits) may be used. The error of each multiplier is now bounded by $\overline{\epsilon_{\text {mult }}}=2^{-p-1-g}$. It can be made arbitrarily small by increasing $g$. Therefore, there exists some $g$ such that $N \overline{\epsilon_{\text {mult }}}<2^{-p-1}$ Actually, this is true even if a less-than-perfect multiplier architecture is used, as soon as we may compute a bound $\overline{\epsilon_{\text {mult }}}$ of its accuracy, and this bound is proportional to $2^{-g}$.

However, we now have another issue: the intermediate result now has $g$ more bits at its LSB than we need. It therefore needs itself to be rounded to the target format. This is easy, using the identity $\circ(x)=\left\lfloor x+\frac{1}{2}\right\rfloor$ : rounding to precision $2^{-p}$ is obtained by first adding $2^{-p-1}$ (this is a single bit) then discarding bits lower than $2^{-p}$. However, in the worst case, this will entail an error $\epsilon_{\text {final rounding }}$ of at most $2^{-p-1}$.

To sum up, the overall error of a faithful architecture SCP is therefore

$$
\epsilon=\epsilon_{\text {final rounding }}+\sum_{i=0}^{N-1} \epsilon_{i}<2^{-p-1}+N \overline{\epsilon_{\text {mult }}}
$$

and this error can be made smaller than $2^{-p}$ as soon as we are able to build multipliers such that

$$
N \overline{\epsilon_{\text {mult }}}<2^{-p-1} \text {. }
$$

All the previous was quite independent of the target technology: it could apply to ASIC synthesis as well as FPGA. Also, a very similar analysis can be developed for an innerproduct architecture where the $a_{i}$ are not constant. Conversely, the following is more focused on a particular context: LUTbased SCP architectures for FPGAs. It explores architectural 


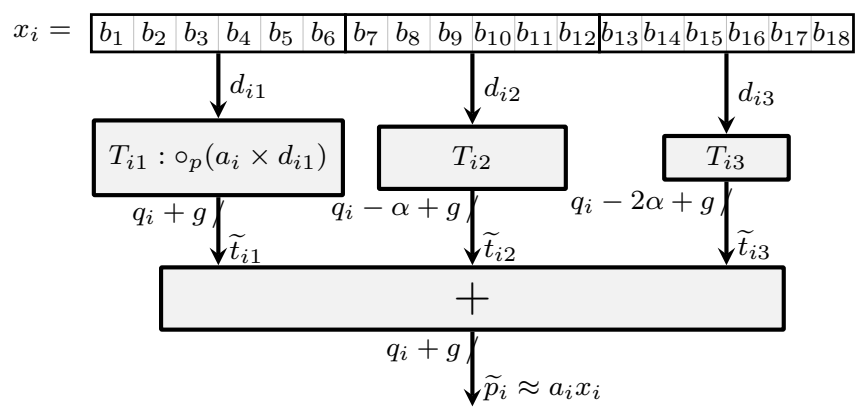

Fig. 3. The FixRealKCM method when $x_{i}$ is split in 3 chunks

means to reach last-bit accuracy at the smallest possible cost, what we call "computing just right" in the title.

\section{AN EXAMPLE OF ARCHITECTURE FOR FPGAS}

On most FPGAs, the basic logic element is the look-uptable (LUT), a small memory addressed by $\alpha$ bits. On the current generation of FPGAs, $\alpha=6$.

\section{A. Perfectly rounded constant multipliers}

As we have a finite number of possible values for $x_{i}$, it is possible to build a perfectly rounded multipliers by simply tabulating all the possible products. The precomputation of table values must be performed with large enough accuracy (using multiple-precision software) to ensure the correct rounding of each entry. This even makes perfect sense for small input precisions on recent FPGAs: if $x_{i}$ is a 6-bit number, each output bit of the perfectly rounded product $a_{i} x_{i}$ will consume exactly one 6-input LUTs. For 8-bit inputs, each bit consumes only 4 LUTs. In general, for $(6+k)$-bit inputs, each output bit consumes $2^{k}$ 6-LUTs: this approach scales poorly to larger inputs. However, perfect rounding to $p+g$ bits means a maximum error smaller than an half-LSB: $\overline{\epsilon_{\text {mult }}}=2^{-p-g-1}$. Note that for real-valued $a_{i}$, this is more accurate than using a perfectly rounded multiplier that inputs $\circ_{p}\left(a_{i}\right)$ : this would accumulate two successive rounding errors.

\section{B. Table-based constant multipliers for FPGAs}

For larger precisions, we may use a variation of the KCM technique, due to Chapman [1] and further studied by Wirthlin [2]. The original KCM method addresses the multiplication by an integer constant. We here present a variation that performs the multiplication by a real constant.

This method consists in breaking down the binary decomposition of an input $x_{i}$ into $n$ chunks $d_{i k}$ of $\alpha$ bits. With the input size being $m+f$, we have $n=\lceil(m+f) / \alpha\rceil$ such chunks ( $n=3$ on Figure 3). Mathematically, this is written

$$
x_{i}=\sum_{k=1}^{n} 2^{-k \alpha} d_{i k} \quad \text { where } \quad d_{i k} \in\left\{0, \ldots, 2^{\alpha}-1\right\} .
$$

The product becomes

$$
a_{i} x_{i}=\sum_{k=1}^{n} 2^{-k \alpha} a_{i} d_{i k}
$$

$$
\begin{aligned}
& \tilde{t}_{i 1}=o_{p}\left(a_{i} d_{i 1}\right) \\
& \tilde{t}_{i 2}=o_{p}\left(a_{i} d_{i 2}\right) \\
& \tilde{t}_{i 3}=\circ_{p}\left(a_{i} d_{i 3}\right)
\end{aligned}
$$

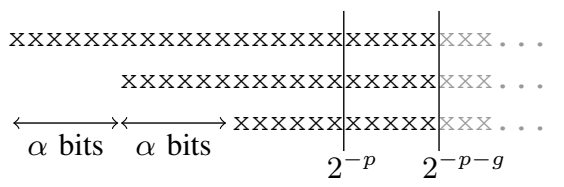

Fig. 4. Aligment of the terms in the KCM method

Since each chunk $d_{i k}$ consists of $\alpha$ bits, where $\alpha$ is the LUT input size, we may tabulate each product $a_{i} d_{i k}$ in a look-up table that will consume exactly one $\alpha$-bit LUT per output bit. This is depicted on Figure 3. Of course, $a_{i} d_{i k}$ has an infinite number of bit in the general case: as previously, we will round it to precision $2^{-p-g}$. In all the following, we define $\widetilde{t}_{i k}=$ ${ }^{\circ} p\left(a_{i} d_{i k}\right)$ this rounded value (see Figure 4).

Contrary to classical (integer) $\mathrm{KCM}$, all the tables do not consume the same amount of resources. The factor $2^{-k \alpha}$ in (9) shifts the MSB of the table output $\widetilde{t}_{i k}$, as illustrated by Figure 4.

Here also, the fixed-point addition is errorless. The error of such a multiplier therefore is the sum of the errors of the $n$ tables, each perfectly rounded:

$$
\epsilon_{\text {mult }}<n \times 2^{-p-g-1} .
$$

This error is proportional to $2^{-g}$, so can made as small as needed by increasing $g$.

\section{Computing the sum}

In FPGAs, each bit of an adder also consumes one LUT. Therefore, in a KCM architecture, the LUT cost of the summation is expected to be roughly proportional to that of the tables. However, this can be improved by stepping back and considering the summation at the SPC level. Indeed, our faithful SPC result is now obtained by computing a double sum:

$$
\widetilde{y}=o_{p}\left(\sum_{i=0}^{N-1} \sum_{k=1}^{n} 2^{-k \alpha} \widetilde{t}_{i k}\right)
$$

Using the associativity of fixed-point addition, this summation can be implemented very efficiently using compression techniques developed for multipliers [6] and more recently applied to sums of products [7], [8]. In FloPoCo, we may use the bit-heap framework introduced in [4]. Each table throws its $\widetilde{t}_{i k}$ to a bit-heap that is in charge of performing the final summation. The bit-heap framework is naturally suited to adding terms with various MSBs, as is the case here.

This is illustrated on Fig. 5, which shows the bit heaps for two classical filters from [5]. On these figures, we have binary weights on the horizontal axis, and the various terms to add on the vertical axis. These figure are generated by FloPoCo before bit heap compression.

We can see that the shape of the bit heap reflects the various MSBs of the $a_{i}$. One bit heap is higher than the other one, although they add the same number of product and each product should be decomposed into the same number of $\mathrm{KCM}$ tables. This is due to special coefficient values that lead to specific optimizations. For instance $a_{i}=1$ or $a_{i}=0.5$ lead to a single addition of $x_{i}$ to the bit heap; $a_{i}=0$ leads to nothing. 


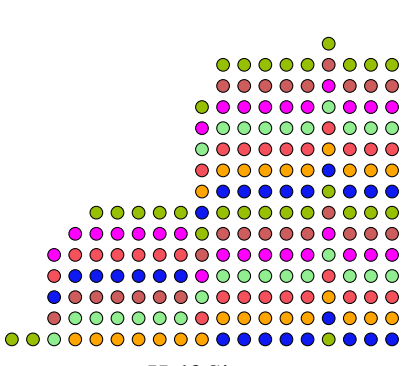

Half-Sine

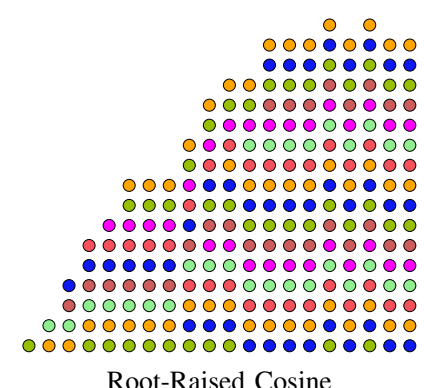

Root-Raised Cosine

Fig. 5. Bit heaps for two 8-tap, 12-bit FIR filters generated for Virtex-6

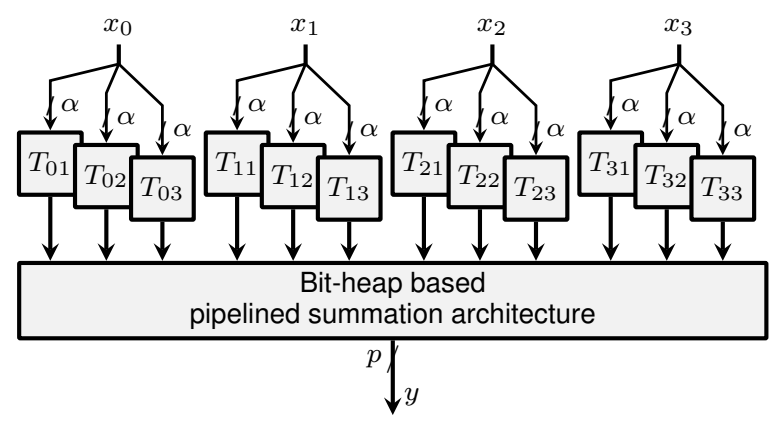

Fig. 6. KCM-based SPC architecture for $N=4$, each input being split into 3 chunks

\section{Managing the constant bits}

Actually there are two more terms to add to the summation of Eq. (11): the rounding bit $2^{-p-1}$, necessary for the final rounding-by-truncation as explained in Section III-C, and the sum of all the sign-extension constants introduced in Section III-A. These bits, especially the rounding bit, are visible on Fig. 5.

There is a minor optimization to do here: these constant bits form a binary constant that can be added (at table-filling time) to all the values of one of the tables, for instance $T_{00}$. Then the rounding bit will be added for free. The sign extension constants may add a few MSB bits to the table output, hence increase its cost by a few LUTs. Still, with this trick, the summation to perform is indeed given by Eq. (11), and the final rounding, being a simple truncation, is for free. This optimization will be integrated to our implementation before publication.

Finally, the typical architecture generated by our tool is depicted by Figure 6.

\section{E. Pipelining}

In terms of speed, reading the table values takes one LUT delay, this is as fast as it gets on an FPGA. The summation, however, may have a significant delay - at most that of $N-1$ additions, but much less in practice thanks to efficient compressor architectures on recent FPGAs. In any cases, the BitHeap framework takes care of the pipelining.

For instance, when using the previous to implement a FIR, we obtain the architecture of Fig. 7. Pipelining the summation may add some latency to the operator, especially compared to an inverted design. However, the bit-heap saves area by

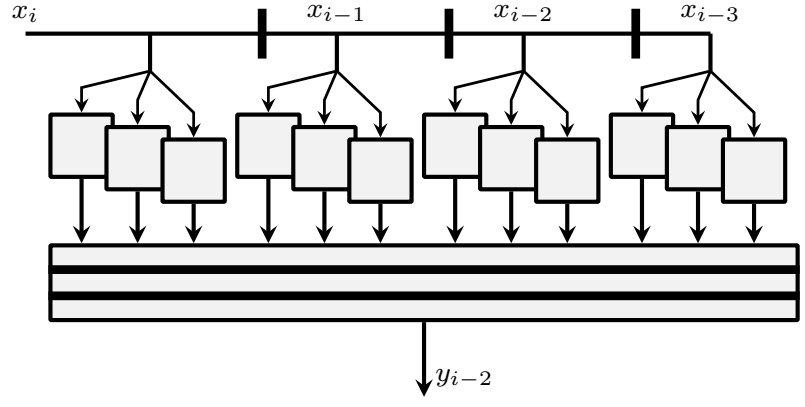

Fig. 7. A pipelined FIR - thick lines denote pipeline levels

\begin{tabular}{|c|c||c|cc|}
\hline Taps & I/O size & Speed & \multicolumn{2}{c|}{ Area } \\
\hline \hline 8 & 12 bits & $\begin{array}{c}4.4 \mathrm{~ns}(227 \mathrm{Mhz}) \\
1 \text { cycle @ 344 Mhz }\end{array}$ & $\begin{array}{l}\text { 564 LUT } \\
\text { 594 LUT }\end{array}$ & 32 Reg. \\
\hline \hline 8 & 18 bits & $\begin{array}{c}5.43 \mathrm{~ns}(184 \mathrm{MHz}) \\
2 \text { cycles @ 318 MHz }\end{array}$ & $\begin{array}{l}\text { 1325 LUT } \\
\text { 1342 LUT }\end{array}$ & 92Reg. \\
\hline \hline 16 & 12 bits & $\begin{array}{c}5.8 \mathrm{~ns}(172 \mathrm{Mhz}) \\
1 \text { cycle @ 289 Mhz }\end{array}$ & $\begin{array}{l}\text { 1261 LUT } \\
\text { 1257 LUT }\end{array} \quad$ 41 Reg. \\
\hline \hline 16 & 18 bits & $\begin{array}{c}7.3 \mathrm{~ns}(137 \mathrm{MHz}) \\
2 \text { cycles @ 265 MHz }\end{array}$ & $\begin{array}{l}\text { 2863 LUT } \\
\text { 2810 LUT }\end{array}$ 120 Reg. \\
\hline \multicolumn{4}{|c|}{ Note: the register count does not include the input shift register. }
\end{tabular}

TABLE I. SyNTHESIS RESUltS OF A RoOT-RAISED COSINE FIR.

optimizing the summation globally, instead of considering as a sequence of additions.

\section{IMPLEMENTATION AND RESULTS}

The method described in this paper is implemented as the FixFIR operator of FloPoCo. FixFIR offers the interface shown on Fig. 1, and inputs the $a_{i}$ as arbitrary-precision numbers. On top of it, the operators FixHS and FixRRCF simply evaluate the $a_{i}$ using the textbook formula for HalfSine and Root-Raised Cosine respectively, and feed them to FixFIR. We expect many more such wrappers could be written in the future.

FixFIR, like most FloPoCo operators, was designed with a testbench generator [9]. All these operators reported here have been checked for last-bit accuracy by extensive simulation.

Table I reports synthesis results for architectures generated by FixRRCF, as of FloPoCo SVN revision 2666. All the results were obtained for Virtex-6 (6vhx380tff1923-3) using ISE 14.7. These results are expected to improve in the future, as the (still new) bit-heap framework is tuned in FloPoCo.

\section{A. Analysis of the results}

As expected, the dependency of the area to the number of taps is almost linear. On the one hand, more taps increase the number of needed guard bits. On the other hand, more taps mean a larger bit heap which exposes more opportunities for efficient compression. However, there is also the dependency of the $a_{i}$ themselves to the number of taps.

Conversely, the dependency of area on precision is more than linear. It is actually expected to be almost quadratic, as can be observed by evaluating the number of bits tabulated for each multiplier (see Fig. 4). 
Interestingly, the dependency of the delay to the number of taps is clearly sub-linear. This is obvious from Fig. 6. All the table accesses are performed in parallel, and the delay is dominated by the compression delay, which is logarithmic in the bit-heap height [10].

\section{B. Comparison to a naive approach}

In order to evaluate the cost of computing just right, we built a variant of the FixFIR operator following a more classical approach. In this variant, we first quantize all the coefficients to precision $p$ (i.e such that their LSB has weight $2^{-p}$ ). We obtain fixed-point constants, and we then build an architecture that multiplies these constants with the inputs using the classical (integer) $\mathrm{KCM}$ algorithm.

A simple error analysis shows that the mere quantization to precision $p$ is already responsible for the loss of two bits of accuracy on $\widetilde{y}$. The exact error due to coefficient quantization depends on the coefficients themselves but space is missing here to detail that. Then, the multiplications are exact. However, as illustrated by Figure 8, their exact results extend well below precision $2^{p}$ : they have to be rounded. We just truncate the output of each $\mathrm{KCM}$ operators to $2^{-p}$. This more than doubles the overall error. It also allows the synthesis tools to optimize out the rightmost 6 bits of Fig. 8. Then these truncated products are summed using a sequence of $N-1$ adders of precision $p$.

The results are visible in Table II. The naive approach leads to a much smaller, but slower design. The area difference doesn't come from the tables, if we compare Fig. 4 and Fig. 8. It comes from the summation, which is performed on $g$ more bits in the last-bit accurate approach. Besides, only the lastbit accurate approach uses a bit heap, whose compression heuristics are currently optimized for speed more than for area.

However, this comparison is essentially meaningless, since the two architectures are not functionally equivalent: The proposed approach is accurate to 12 bits, while the naive approach loses more than 3 bits to quantization and truncation. Therefore, a meaningful comparison is the proposed approach, but accurate to $p=9$, which we also show in Table II. This version is better than the naive one in both area and speed.

Since we are comparing two designs by ourselves, it is always possible to dispute that we deliberately sabotaged our naive version. This is right, in a sense. We designed it as small as possible (no guard bits, truncation instead of rounding, etc). Short of designing a filter that returns always zero (very poor accuracy, but very cheap indeed), this is the best we can do. But all these choices are highly disputable, in particular because they impact accuracy.

All this merely illustrates the point we want to make: it only makes sense to compare designs of equivalent accuracies. And the only accuracy that makes sense is last-bit accuracy, for the reasons exposed in II-B. We indeed put our best effort in designing the last-bit accurate solution, and we indeed looked for the most economical way of achieving a given accuracy. This is comforted by this comparison. Focussing on last bit accuracy enables us to compute right, and just right.

For the sake of completeness, we also implemented a version using DSP blocks, reported in Table II. Considering

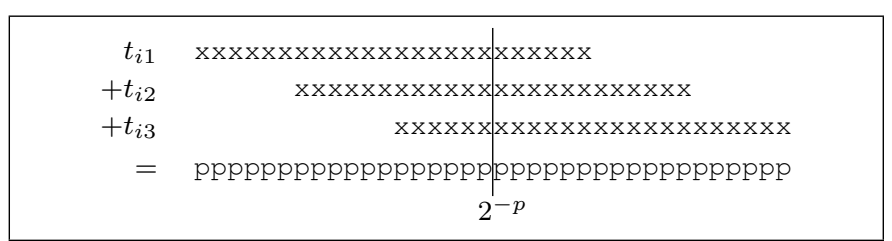

Fig. 8. Using integer KCM: $t_{i k}=o_{p}\left(a_{i}\right) \times d_{i k}$. This multiplier is both wasteful, and not accurate enough.

\begin{tabular}{|l||c|c|c|}
\hline Method & Speed & Area & accuracy \\
\hline \hline$p=12$, proposed & $4.4 \mathrm{~ns}(227 \mathrm{Mhz})$ & 564 LUT & $\bar{\epsilon}<2^{-12}$ \\
\hline$p=12$, naive & $5.9 \mathrm{~ns}(170 \mathrm{Mhz})$ & 444 LUT & $\bar{\epsilon}>2^{-9}$ \\
\hline$p=9$, proposed & $4.12 \mathrm{~ns}(243 \mathrm{Mhz})$ & 380 LUT & $\bar{\epsilon}<2^{-9}$ \\
\hline \hline$p=12$, DSP-based & $9.1 \mathrm{~ns}(110 \mathrm{Mhz})$ & 153 LUT, 7 DSP & $\bar{\epsilon}<2^{-9}$ \\
\hline
\end{tabular}

TABLE II. THE ACCURACY/PERFORMANCE TRADE-OFF ON 8-TAP, 12 BIT ROOT-RAISED COSINE FIR FILTERS.

the large internal precision of DSP blocks, it costs close to nothing to design this version as last-bit accurate for $p=12$ or $p=18$ (in the latter case, provided the constant is input with enough guard bits on the 24-bit input of the DSP block). This is very preliminary work, however. We should offer the choice between either an adder tree with a shorter delay, or a long DSP chain which would be slow, but consume no LUT. We should also compare to Xilinx CoreGen FIR compiler, which generates only DSP-based architectures. Interestingly, this tool requires the user to quantize the coefficients and to take all sorts of decisions about the intermediate accuracies and rounding modes.

\section{CONCLUSION}

This paper claims that sum-of-product architectures should be last bit accurate, and demonstrates that this has two positive consequences: It gives a much clearer view on the trade-off between accuracy and performance, freeing the designer from several difficult choices. It actually leads to better solutions by enabling a "computing just right" philosophy. All this is demonstrated on an actual open-source tool that offers the highest-level interface.

Future work include several technical improvements to the current implementation, such as the exploitation of symmetries in the coefficients or optimization of the bit heap compression.

Beyond that, this work opens many perspectives.

As we have seen, fixed-point sum of products and sum of squares could be optimized for last-bit accuracy using the same approach.

We have only studied one small corner of the vast literature about filter architecture design. Many other successful approaches exist, in particular those based on multiple constant multiplication (MCM) using the transpose form (where the registers are on the output path). [11], [12], [13], [14], [15]. A technique called Distributed Arithmetic, which predates FPGA [16], can be considered a generalization of the KCM technique to the MCM problem. From the abstract of [17] that, among other things, compares these two approaches, "if the input word size is greater than approximately half the number of coefficients, the LUT based multiplication scheme needs less resources than the DA architecture and vice versa". Such a 
rule of thumb (which of course depends on the coefficients themselves) should be reassessed with architectures computing just right on each side. Most of this vast literature treats accuracy after the fact, as an issue orthogonal to architecture design.

A repository of FIR benchmarks exists, precisely for the purpose of comparing FIR implementations [18]. Unfortunately, the coefficients there are already quantized, which prevents a meaningful comparison with our approach. Few of the publications they mention report accuracy results. However, cooperation with this group should be sought to improve on this.

We have only considered here the implementation of a filter once the $a_{i}$ are given. Approximation algorithms, such as Parks-McClellan, that compute these coefficients, essentially work in the real domain. The question they answer is "what is the best filter with real coefficients that matches this specification". It is legitimate to wonder if asking the question: "what is the best filter with low-precision coefficients" could not lead to a better result.

Still in filter design, the approach presented here should be extended to infinite impulse response (IIR) filters. There, a simple worst-case analysis (as we did for SPC) doesn't work due to the infinite accumulation of error terms. However, as soon as the filter is stable (i.e. its output doesn't diverge), it should be possible to derive a bound on the accumulation of rounding errors. This would be enough to design last-bit accurate IIR filters computing just right.

\section{REFERENCES}

[1] K. Chapman, "Fast integer multipliers fit in FPGAs (EDN 1993 design idea winner)," EDN magazine, no. 10, p. 80, May 1993.

[2] M. Wirthlin, "Constant coefficient multiplication using look-up tables," Journal of VLSI Signal Processing, vol. 36, no. 1, pp. 7-15, 2004.

[3] F. de Dinechin, H. Takeugming, and J.-M. Tanguy, "A 128-tap complex FIR filter processing 20 giga-samples/s in a single FPGA," in 44th Asilomar Conference on Signals, Systems \& Computers, 2010.

[4] N. Brunie, F. de Dinechin, M. Istoan, G. Sergent, K. Illyes, and B. Popa, "Arithmetic core generation using bit heaps," in Field-Programmable Logic and Applications, Sep. 2013.

[5] IEEE Std 802.15.4-2006, IEEE Standard for Information technologyTelecommunications and information exchange between systems- Local and metropolitan area networks- Specific requirements- Part 15.4: Wireless Medium Access Control (MAC) and Physical Layer (PHY) Specifications for Low-Rate Wireless Personal Area Networks (WPANs), 2006

[6] M. D. Ercegovac and T. Lang, Digital Arithmetic. Morgan Kaufmann, 2003.

[7] H. Parendeh-Afshar, A. Neogy, P. Brisk, and P. Ienne, "Compressor tree synthesis on commercial high-performance FPGAs," ACM Transactions on Reconfigurable Technology and Systems, vol. 4, no. 4, 2011.

[8] R. Kumar, A. Mandal, and S. P. Khatri, "An efficient arithmetic sum-ofproduct (SOP) based multiplication approach for FIR filters and DFT," in International Conference on Computer Design (ICCD). IEEE, Sep. 2012, pp. 195-200

[9] F. de Dinechin and B. Pasca, "Designing custom arithmetic data paths with FloPoCo," IEEE Design \& Test of Computers, vol. 28, no. 4, pp. 18-27, Jul. 2011. [Online]. Available: http: //ieeexplore.ieee.org/xpls/abs_all.jsp?arnumber $=5753874$

[10] L. Dadda, "Some schemes for parallel multipliers," Alta Frequenza, vol. 34, pp. 349-356, 1965.
[11] M. Potkonjak, M. Srivastava, and A. Chandrakasan, "Efficient substitution of multiple constant multiplications by shifts and additions using iterative pairwise matching," in ACM IEEE Design Automation Conference, San Diego, CA USA, 1994, pp. 189-194.

[12] N. Boullis and A. Tisserand, "Some optimizations of hardware multiplication by constant matrices," IEEE Transactions on Computers, vol. 54, no. 10, pp. 1271-1282, 2005.

[13] M. Mehendale, S. D.Sherlekar, and G. Venkatesh, "Synthesis of multiplier-less FIR filters with minimum number of additions," in IEEE/ACM International Conference on Computer-Aided Design, San Jose, CA USA, 1995, pp. 668-671.

[14] Y. Voronenko and M. Püschel, "Multiplierless multiple constant multiplication," ACM Trans. Algorithms, vol. 3, no. 2, 2007.

[15] L. Aksoy, E. Costa, P. Flores, and J. Monteiro, "Exact and approximate algorithms for the optimization of area and delay in multiple constant multiplications," IEEE Transactions on Computer-Aided Design of Integrated Circuits and Systems, vol. 27, no. 6, pp. 1013-1026, 2008.

[16] S. White, "Applications of distributed arithmetic to digital signal processing: A tutorial review," IEEE ASSP Magazine, no. 3, pp. 4-19, Jul. 1989.

[17] M. Kumm, K. Möller, and P. Zipf, "Dynamically reconfigurable FIR filter architectures with fast reconfiguration," in Reconfigurable and Communication-Centric Systems-on-Chip (ReCoSoC). IEEE, Jul. 2013.

[18] "FIR suite," http://www.firsuite.net/. 